\title{
A narrative review of the role of necroptosis in liver disease: a double-edged sword
}

\author{
Xuehui Li $^{1}$, Guanjun Dong ${ }^{2}$, Huabao Xiong ${ }^{2}$, Hongyan Diao ${ }^{1}$ \\ ${ }^{1}$ State Key Laboratory for Diagnosis and Treatment of Infectious Diseases, National Clinical Research Center for Infectious Diseases, Collaborative \\ Innovation Center for Diagnosis and Treatment of Infectious Diseases, The First Affiliated Hospital, College of Medicine, Zhejiang University, \\ Hangzhou, China; ${ }^{2}$ Institute of Immunology and Molecular Medicine, Jining Medical University, Jining, China \\ Contributions: (I) Conception and design: H Diao, X Li; (II) Administrative support: H Diao, H Xiong; (III) Provision of study materials or patients: \\ H Diao, H Xiong; (IV) Collection and assembly of data: X Li; (V) Data analysis and interpretation: X Li; (VI) Manuscript writing: All authors; (VII) \\ Final approval of manuscript: All authors. \\ Correspondence to: Hongyan Diao. State Key Laboratory for Diagnosis and Treatment of Infectious Diseases, The First Affiliated Hospital, 79 \\ Qingchun Road, Hangzhou 310000, China. Email: diaohy@zju.edu.cn; Huabao Xiong. Institute of Immunology and Molecular Medicine, Jining \\ Medical University, 133 Hehua Road, Jining 273100, China. Email: xionghbl@yahoo.com.
}

\begin{abstract}
Acute and chronic liver injuries lead to hepatocyte death and turnover. When injuries become chronic, continuous cell death and transformation lead to chronic inflammation, fibrosis, cirrhosis, and eventually carcinoma. A therapeutic strategy of great significance for liver disease is to control hepatocyte death in acute and chronic injuries. This strategy prevents hepatocytes from causing liver failure and inhibits both secondary inflammation and fibrosis. Both apoptosis and necrosis have been proven to occur in the liver, but the role of necroptosis in liver diseases is controversial. Necroptosis, which has features of necrosis and apoptosis, is a regulatory process that occurs in some cell types when caspases are inhibited. The signaling pathway of necroptosis is characterized by the activation of receptor-interacting proteins kinase (RIPK) and mixed lineage kinase domain-like (MLKL). Necroptosis is associated with a variety of inflammatory diseases and has been the focus of research in recent years. The incidence of necroptosis in liver tissues has been studied recently in several liver injury models, but the results of the studies are not consistent. The purpose of this review is to summarize the published data on the involvement of necroptosis in liver injury, focusing on the controversies, issues remaining to be discussed, and potential therapeutic applications in this area.
\end{abstract}

Keywords: Necroptosis; acute liver disease; chronic liver disease; liver cancer

Submitted Jul 06, 2020. Accepted for publication Jan 15, 2021.

doi: $10.21037 /$ atm-20-5162

View this article at: http://dx.doi.org/10.21037/atm-20-5162

\section{Introduction}

Liver disease is a major reason for illness and death worldwide. The burden of liver diseases in China is primarily due to viral hepatitis, nonalcoholic fatty liver disease, and alcoholic liver disease (1). Although many preventative measures have been adopted over the years, the number of patients with alcoholic and nonalcoholic fatty liver diseases is still increasing rapidly. Liver cancer is one of the most fatal cancers in the world and the second-most common cancer in China. The prognosis of hepatocellular carcinoma (HCC) is extremely poor due to its insidious onset, invasiveness, aggressiveness, high recurrence rate, and fatality. The HCC 5-year average survival rate is less than $10 \%$ (2). Liver disease remains a major threat to public health, human health, and quality of life.

Necroptosis is a newly discovered pathway of regulated necrosis that is mediated by receptor-interacting proteins kinase 1 and 3 (RIPK1, RIPK3) and MLKL (3-5). It is induced by death receptors, interferons, toll-like receptors (TLRs), intracellular RNA and DNA sensors, and probably 
other mediators as well (6). Typically, necroptosis is regarded as a pro-inflammatory mode of cell death that releases intracellular components. However, many studies have shown that necroptosis may have anti-inflammatory effects by blocking the excessive production of chemokines $(7,8)$. It has already been shown that RIPKs are involved in multiple pathophysiologies, and the inhibition of these kinases could have therapeutic potentials $(9,10)$. However, the contribution of caspase-independent RIPK 3 and MLKL to hepatocyte necroptosis remains debatable (11). However, it has become evident that, other than apoptosis, necroptosis is also a highly relevant form of programmed cell death (PCD) in the liver (12). The minimal expression of a key player involved in necroptosis in the liver suggests that it may be uncommon in liver diseases (13). In order to find new and effective targets for the treatment of liver diseases, it is essential to understand the role of necroptosis at different stages of liver disease to develop new therapeutic approaches. In this review, we summarize the recent progress in identifying the role of necroptosis in the pathogenesis of liver diseases. We present the following article in accordance with the Narrative Review reporting checklist (available at http://dx.doi.org/10.21037/atm-20-5162).

\section{Characteristics and classical signaling pathway of necroptosis}

Necrosis and apoptosis are well-established forms of cell death, that occurs through various physiological and pathological processes. In addition to these, many new means of cell death have been discovered and characterized, including necroptosis, pyroptosis, and ferroptosis. Necroptosis first came to light in 1996 and was officially termed in $2005(14,15)$. Many factors contribute to necroptosis, such as microbial infections and physicochemical stressors. However, no specific necroptotic damage-associated molecular patterns (DAMPs) have been found (10). Necroptosis is a genetically programmed and caspase-independent form of cell death (16). The signaling molecules that are considered to be responsible for the activation of necroptosis are RIPK1, RIPK3, and MLKL (17-19). Necroptosis, pyroptosis, and apoptosis share components of a common signaling pathway. Morphologically, necroptosis is similar to necrosis. It is characterized by cellular and organelle swelling, membrane damage, and release of intracellular contents, which all contribute to inflammatory reactions in lesion areas $(20,21)$.

The tumor necrosis factor (TNF) and TNF receptor 1 (TNFR1)-mediated signaling pathway is one of the most extensively studied models of necroptosis (22). It is prevalent in different types of tumors and other pathophysiological conditions (23). This classical signaling pathway is initiated by the binding of the TNF ligand to its receptor TNFR1, which coalesce and provide a binding site for TNFR1-associated death domain protein (TRADD) and the polyubiquitinated receptor-interacting protein 1 (RIP1). After RIP1 is deubiquitinated by cylindromatosis (CYLD), caspase 8, RIP1, RIP3, and Fas-associated protein with death domain (FADD) form complex IIa. Apoptosis occurs when caspase 8 is activated. When the activity of caspase 8 is inhibited (such as through zVAD), RIP1 and RIP3 do not separate from each other and form a necrosome (complex IIb). The necrosome, composed of RIP1, RIP3, and MLKL, is phosphorylated in a stepwise manner. Phosphorylated MLKL is then transported into the nucleus and plasma membrane, triggering membrane punctures and cell rupture, and thereby leading to necroptosis $(24,25)$. Despite the critical role of RIP1 in necroptosis, and researches suggest that there are RIP1independent necroptosis pathways. It has been shown that virus-induced necroptosis depends on the DNAdependent activator interferon-regulatory factor (DAI) and RIP3, independent of RIP1 function (26). Furthermore, interferons (IFNs) can also trigger necroptosis independent of RIP1 via the interferon-induced protein kinase PKR (27). The above relevant signal pathway has been shown in Figure 1. In addition, RIP1 kinase does not contribute to TRIF-dependent necroptosis in some kind of cell types apart from macrophages (26). Multiple molecular inhibitors have emerged as requirements of initiation of necroptosis. Necrostatins, including Nec-1 and Nec-2, inhibit RIPK1; kinase inhibitors, including GSK'840, GSK'843, and GSK'872, inhibit RIPK1 and RIPK3; and necrosulfonamide (NSA) inhibits MLKL (28). In addition, several natural products and isolated compounds, such as curcumin and kongensin $\mathrm{A}$, were found to have an inhibitory effect on necroptosis (12).

Recently, necroptosis has emerged as a critical pathway underlying human diseases (29-31), such as breast cancer (32), acetaminophen-induced-liver injury (33), 


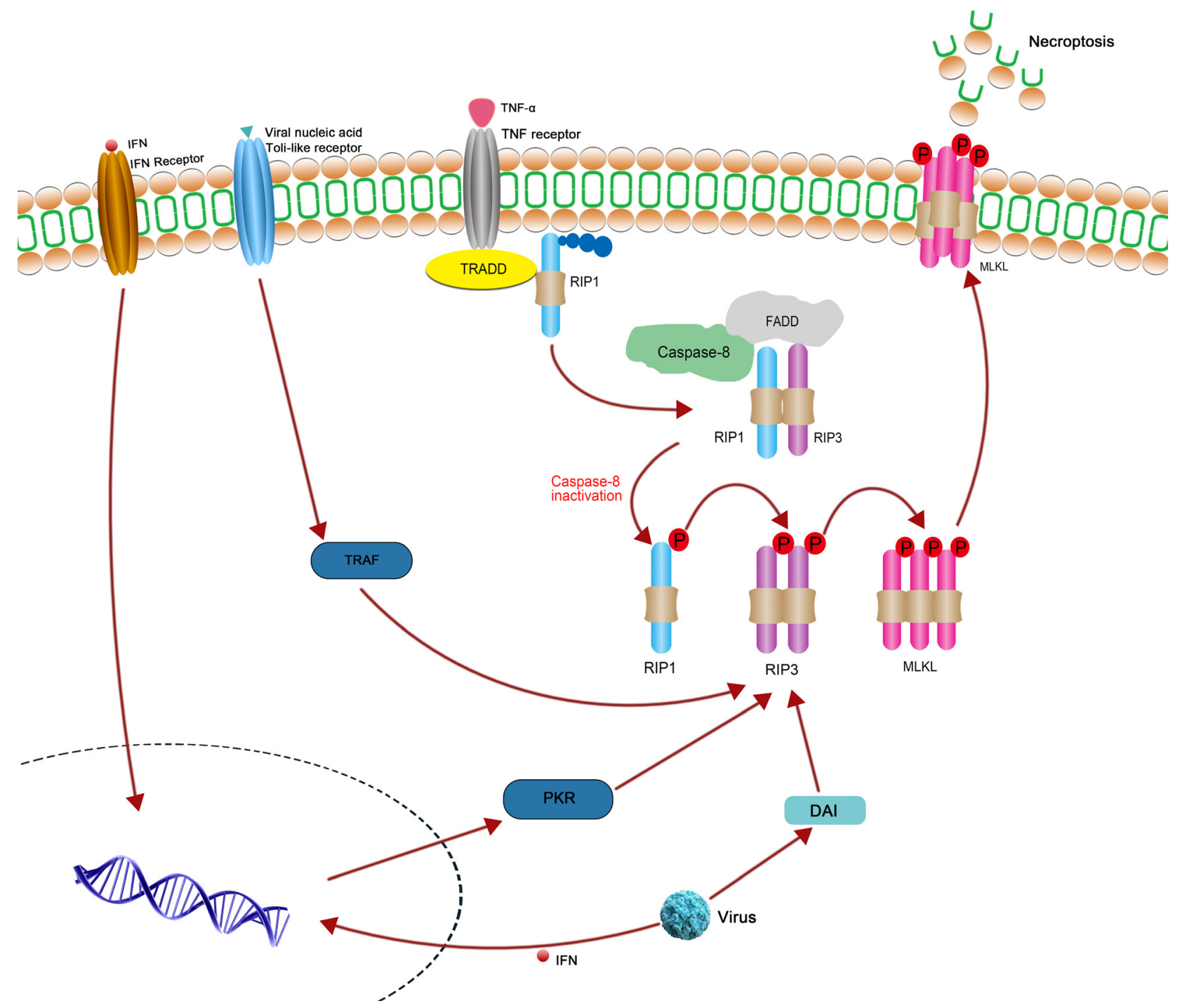

Figure 1 The classical necroptosis signaling pathway initiated by the combining of TNF ligand and TNF receptor 1 . The combination of TNF $\alpha$ and TNF receptor 1 can induce necroptosis, when the caspase- 8 activity is inhibited. The binding between RIP1, RIP3 and MLKL induces the phosphorylation of MLKL and subsequent execution of necroptosis. In addition, IFNs also trigger necroptosis independently of RIPK1 via the interferon-induced protein kinase PKR. Toll receptor and DAI can activate RIPK3 independent of RIPK1.

autoimmune disease, and autoinflammatory disease (29).

\section{Current strategies to detect necroptosis in the liver}

Typically, the activation of RIP1, RIP3 and MLKL causes the occurrence of necroptosis. Despite their potential use as markers, there is still no highly specific test for the immunohistochemical detection of necroptosis (34).
Although western blotting seems to be relatively specific and reliable for the evaluation of RIPK3 expression, the activation of this pathway in parenchymal cells and non-parenchymal cells cannot be clearly distinguished through western blotting (34). The antibodies for p-MLKL are not sensitive enough to detect a subtle activation of MLKL in hepatocytes or livers [for instance in mouse nonalcoholic steatohepatitis (NASH) models]. Furthermore, the phosphorylation of MLKL does not 
necessarily indicate necroptotic cell death, because MLKL can self-phosphorylate. RIPK1/3 have multiple function apart from mediator of necroptosis $(35,36)$. Another marker we have used to detect necroptosis is p-RIPK1. Several autophosphorylation sites of RIP1 have been identified in in vitro kinase assays, including Ser14/15, Ser20, Ser161, and Ser166. Anti-phospho-RIP1 antibodies have been developed as makers for the activation of RIP1, which can specifically recognize the phospho-Ser166 and phosphoSer14/15 of hRIP1 (34). However, both apoptosis and necroptosis are detected because RIPK1 is involved in both signaling pathways (11). Hence, the accurate detection and recognition of necroptosis is difficult. Nevertheless, there are other possible methods to detect necroptosis. For instance, necrosome assembly and RIP3-MLKL have been shown to interact through co-immunoprecipitation experiments or microscopically using the DuoLink ${ }^{\circledR}$ proximity ligation assay (37-39). The aggregation status of RIP3 and MLKL can also be monitored by evaluating their retention in insoluble/soluble protein fractions, or by co-staining of thioflavin $\mathrm{T}$, a $\beta$-amyloid fibril marker, with RIP3 puncta (40-42).

Thus, definitive identification of necroptosis requires affirmation by different detection methods and by detection of several kinds of signaling molecular, such as combining the biochemical and morphological tests. Up to now, immunohistochemistry and western blot analyses for phosphor-MLKL are considered acceptable methods to detect necroptosis $(41,43)$.

\section{Necroptosis as a potential target for the treatment of liver disease}

Recently, the pathophysiological role of necroptosis has been highlighted in inflammatory diseases and it has become an attractive target for the treatment of liver diseases (40). As RIPK3 is more specific to necroptosis, it is believed that cells that do not express RIPK3 do not undergo necroptosis. The occurrence of necroptosis in the liver and its contribution to liver diseases is debated. Because RIPK3 is difficult to be detected in hepatocytes under basal conditions (44). Additionally, evidence has shown that RIPK3 is predominantly expressed in nonparenchymal cell fractions (non-LPCs) (45). Accordingly, some researchers believe that cholangiocytes can undergo necroptosis, while primary hepatocytes die exclusively by apoptosis after treating with cell death-inducing stimuli in vitro (45). Other research proved that caspase 8 competent wild-type hepatocytes express RIPK3 at low levels compared with many other cell types, but it is sufficient to trigger necroptosis and inflammation when caspase 8 is depleted from hepatocytes in the livers of mice in vivo (12). Further, RIPK3 has been detected in liver biopsies of NASH patients, as well as in the liver of mice exposed to diets deficient in methyl-choline or high in fat, suggesting a role of necroptosis in the pathogenesis of non-alcoholic fatty liver disease (NAFLD)/NASH (46). Moreover, in human patients, necroptosis is activated in alcoholic liver injuries, which are the leading causes of liver cirrhosis and HCC in the western world $(47,48)$. Research shows that RIPK3 mRNA levels in peripheral blood mononuclear cells (PBMCs) are linked to poor prognosis of acute-on-chronic hepatitis $\mathrm{B}$ liver failure (ACHBLF), thus necroptosis is proposed to play a critical role in the pathogenesis of ACHBLF (49). While the transcription of MLKL was very low in healthy controls, MLKL expression in hepatocytes of AIH patients, in patients with steatosis, and patients with primary biliary cirrhosis was strongly upregulated (50). When infected by Listeria monocytogenes, Kupffer cells residing in the liver underwent necroptotic cell death, which therefore balances type 1 microbicidal inflammation and type-2-mediated liver repair upon infection (51). According to a relevant study, RIPK3 is involved in cytokine production of liver leukocytes after being stimulated by $\alpha$-galactosylceramide ( $\alpha$-GalCer) (52). Furthermore, acidic extracellular $\mathrm{pH}(\mathrm{pHe})$ switches TNF-related apoptosisinducing ligand (TRAIL)-induced apoptosis to necroptosis in human HT29 and HepG2 cells (53).

We conclude that in order for primary hepatocytes to execute necroptosis, an additional sensitization step is required. It is possible that under pathological conditions, augmented levels of RIPK3 or MLKL switch apoptosis to necroptosis in cells with low expression of these key necroptotic proteins. Furthermore, important functions of necroptosis might exist in other cell types, such as biliary cells, hepatic stellate cells, Kupffer cells or endothelial cells $(12,51)$. In summary, necroptosis plays a critical role in liver diseases.

\section{The role of necroptosis is still incompletely defined in hepatitis, cirrhosis, and liver cancer}

Acute liver failure is characterized by massive loss of parenchymal cells but is usually followed by restitution and integrum $(12,50)$. By contrast, in chronic liver diseases, hepatocytes degenerate, necrose, and regenerate. 
Meanwhile, fibrous tissues proliferate and repair, ultimately leading to cirrhosis and HCC. Necroptosis takes place during hepatic regeneration, inflammation and fibrogenesis in the hepatic disease condition (54). To gain a deeper understanding of the relationship between necroptosis and liver diseases, we review on the role of necroptosis at different stages of liver diseases.

\section{Necroptosis promotes inflammation in acute liver disease}

Acute liver inflammation is usually induced by bacterial infection or physical and chemical injury. It is reported that higher necroptosis of hepatocytes emerged in Gal-3 deficient mice in acute $\mathrm{CMV}$-induced liver disease (55). In patients suffering from drug-induced liver injury (DILI), cell death was demonstrated to be associated with activation of MLKL (56). In fact, there are many controversies arising from the use of acetaminophen (APAP)-overdosed mouse models. Generally, we believe that inhibition of RIP1 or RIP3 can alleviate APAP-induced acute liver injury, but the role of MLKL in APAP-induced acute liver injury is still uncertain (57-60). In immune mediated acute liver injury models, such as those induced by concanavalin A (Con-A) and $\alpha$-GalCer, Sib-RIPK1 inhibitor protects mice from Con A-induced hepatitis (61). Nec-1was found to reduce liver cell death and alleviate liver damage in $\mathrm{Fmr}^{\text {null }}$ mice following TNF exposure. Deletion of RIPK3 delays hepatic injury induced by Con-A (58). Consistent with these findings, increased pathology after bile duct ligation coincided with increased hepatic expression of RIPK1, RIPK3 and p-MLKL $(41,62,63)$. Similarly, pretreatment with Nec-1 or RIPK3 deletion protect against ConA and $\alpha$-GalCer induced liver injury (44). This protection against ConA-induced liver injury is associated with downregulated hepatocyte-specific IL-33 expression. It should be noted that IL-33 is a classical DAMP that is tightly linked to necrosis or necroptosis (64). Apart from this, deletion of RIPK3 and pharmacological inhibition of dynamin related protein 1 (Drp1) protect mice from natural killer $\mathrm{T}$ (NKT) cell-mediated induction of acute liver damage. Jouan-Lanhouet et al. suggest that use of pharmacological inhibitors of RIPKs could be a new strategy for treatment of immune-mediated hepatitis (53). These findings suggest that necroptosis may promote inflammation in acute liver injury.

However, mice expressing a form of RIPK1 lacking kinase activity were protected, whereas deletion of RIPK1 in hepatocytes aggravated ConA-induced liver injury.
Even, it is reported that Nec-1 severely exacerbated ConAinduced disease and mice with ablation of RIPK 3 were not protected (58). Combining reports that RIPK3 has also been suggested to regulate NKT cell function and promote the NKT cell mediated anti-tumor immune response through a process that is independent of the necroptosis pathway (52). Apart from this, it is suggested that different components of the necrosome must be considered independently to analyze their opposite effect on damage (58). The protective function of RIPKs was not confirmed in conditional, hepatocytespecific RIPKs knockout mice. Collectively, RIPKs may have other important functions independent of their actions as cell death mediators, and their role in acute liver injury remains poorly understood $(20,44)$. It is reported that RIPK 3 can promote NLRP 3 inflammasome and IL- $1 \beta$ inflammatory responses independent of MLKL and necroptotic cell death (65). Furthermore, in addition to causing necroptosis by rupturing the membrane, MLKL controls the transport of endocytosed proteins, thereby enhancing the degradation of receptors and ligands. Thus, the extracellular release of phosphorylated MLKL within vesicles serves as a mechanism for self-restricting the necroptotic activity of this protein (66). Wong et al. showed that RIPK1 and RIPK3 can facilitate the production of multiple cytokines, independent of their role in necroptosis (67). They further found that the kinase activities of RIPK1 and RIPK3, in addition to their roles in regulating necroptosis, have a physiological role in the tumor microenvironment, in particular tumor cell extravasation and remodeling by altering the downstream signaling pathways of permeability factors (68).

\section{Inbibiting necroptosis can alleviate chronic liver disease and cirrbosis}

Chronic liver inflammation (CLI), a complex process, exhibits progressive destruction and regeneration of liver parenchymal cells (LPCs). There are a multitude of reasons for CLI, such as viral infection, alcohol abuse, drug toxicity, and genetic metabolic disorders (69). Chronic pathologic processes mainly include viral infection, alcoholic liver disease (ALD), NASH, and autoimmune diseases, which lead to liver cirrhosis and ultimately HCC (69). Hepatocytes suffer a variety of forms of death including apoptosis, necrosis and necroptosis during the CLI progression. In recent years, several studies have shown that RIPK3-dependent necroptosis is an important pathway that regulates fibrosis progression (12,70-73). 
It was reported that the inflammation and liver pathology decreased in vaccinia virus-infected RIPK3-deficient mice with altered TNF $\alpha$-induced necroptosis (74). Moreover, mice infected by vaccinia virus can present the accumulation of the RIPK1/RIPK3 complex in liver (39); both of these results indicate that necroptosis participates in the process of viral infection of the liver. Hepatitis $B$ virus (HBV) and hepatitis $\mathrm{C}$ virus (HCV) are well known as leading causes of liver cirrhosis and HCC (75). The effect of HCV on hepatocyte cell death might be prevented in vitro by inhibitors of apoptosis and necroptosis (76). Moreover, Han et al. showed that RIPK3 mRNA levels were significantly higher in patients with ACHBLF. RIPK3 and MLKL were negativity regulated by caspase- 8 , which was positively correlated with liver injury and poor prognosis (49). They concluded that necroptosis might play an important role in the pathogenesis of this disease.

Chronic alcohol consumption can lead to ALD, which is characterized by steatosis, inflammation, fibrosis, and cirrhosis, and may even cause liver cancer (47). ALD, which features excessive hepatocyte death and inflammation, is a prevalent disease that causes a heavy health burden worldwide. Some studies revealed that hepatocyte necroptosis is a central event that promotes inflammation in ALD (77). Roychowdhury et al. indicated that RIPK3 expression was increased in ethanol-fed mouse livers and in ALD patients' livers. Furthermore, RIPK3-mediated necroptosis is highly correlated with poor prognosis in patients with alcoholic cirrhosis (38,72). Accordingly, ethanol-induced liver injury, steatosis, and inflammation were decreased in RIP3 knockout mice compared to control mice (38). We can conclude that RIPK3 is important in mediating ethanol-induced liver injury and progression of ALD.

With regard to hepatocyte cell death in NASH, apoptosis was regarded as the key driver by most studies. Apoptosis was detected in both liver tissues from patients with NASH and in commonly used mouse models of NASH. However, Gautheron et al. confirmed that blockage of necroptosis via ablation of RIPK3 protected mice from NASH-induced liver fibrosis, and they deduced that necroptosis rather than apoptosis controls liver fibrosis (71). They could not detect the activation of apoptosis in the liver of mice fed with methionine-choline-deficient (MCD), and western blotting showed that RIPK3 expression in this model was strongly up-regulated (40). Similarly, patients with NASH had a dramatic upregulation of hepatic RIPK3 expression as an indicator of necroptosis, suggesting a shift away from apoptotic towards necroptotic signaling in the livers of these patients (40). More importantly, in the MCD NASH mouse model, injury and fibrosis indicated that the main role of caspase- 8 in NASH was to prevent the over-activation of necroptosis. By contrast, inhibition of RIPK3 in the MCD NASH model ameliorated liver injury and fibrosis, providing evidence that necroptosis, not apoptosis, is the driver of liver injury and liver fibrosis in this well-established mouse model of NASH. Along with these findings in mice, a western blot analysis of frozen liver tissue from a cohort of patients with biopsy-proven $\mathrm{NASH}$, showed that these patients had lower levels of intrahepatic caspase 3 cleavage than healthy individuals. In a genetic model of chronic hepatic inflammation, chronic inflammation induced by LPC-specific deletion of tumor growth factor- $\beta$-activated kinase-1 (Tak1), RIPK3-dependent necroptosis represents a pathway. This pathway regulates the consequences of chronic inflammation in the liver by counteracting against caspase 8-dependent compensatory proliferation of hepatocytes, immune cell activation, hepatic fibrogenesis, and the development of chromosomal aberrations leading to hepatocarcinogenesis (78).

NAFLD is the initial stage of NASH, often contributing ultimately to liver fibrosis and liver cancer (71). Studies indicate that necroptosis is a key pathogenic event in human and experimental murine models of non-alcoholic steatohepatitis (40). RIPA-56 was regarded as a highly specific and metabolically stable inhibitor of RIPK1 (79). In a newly published study, Majdi et al. showed that RIPA56 significantly reduced all liver features associated with steatohepatitis, including inflammation and liver fibrosis, in mice with high fat diet (HFD) and hepatocytes from patients with NAFLD (80). They also suggested that necroptosis contributed to the pathogenesis of NAFLD, and that the release of RIPK1 and MLKL into systemic circulation reflects necro-inflammatory activity in human NAFLD. Furthermore, MLKL but not RIPK3 contributes to Western diet-induced liver injury and a murine model of non-alcohol-associated fatty liver and steatohepatitis through inhibition of autophagy and induction of necroptosis $(81,82)$. Even the expression of RIPK3 was upregulated in mice with hepatic steatosis induced by HFD. However, previous reports about the role of RIPK3 in HFD are controversial $(83,84)$. RIP3 deficiency protects from liver disease in MCD-fed mice $(40,71)$, whereas deletion of RIP3 exacerbates liver damage and steatosis, and glucose intolerance induced by a high-fat diet (83). These conflicting results may be due to different modeling 
methods. Compared with liver injury induced by HFD, mice on MCD diet will show more severe lipidosis, fibrosis, and more active oxidative stress. At the same time, there are differences in modeling performance with different fat composition and proportions $(85,86)$. Furthermore, these data imply that RIP1 and RIP3 cannot be simply considered part of a single functional unit. Indeed, much of the literature in acute liver injury is hindered by conflicting reports between laboratories (58).

In a liver fibrosis model induced by $\mathrm{CCl}_{4}, \mathrm{CCl}_{4}$ significantly increased RIPK1 expression, the formation of the RIPK1 and RIPK3 necrosome complex, and the level of MLKL protein in liver tissue, all of which were attenuated by MLT (87). This finding suggested that MLT may prevent liver fibrosis by inhibiting necroptosisassociated inflammatory signaling. A study showed that curcumol protects mice liver from $\mathrm{CCl}_{4}$-induced injury and ameliorates murine hepatic fibrogenesis. The researchers investigated further and eventually found that curcumol reduces the expression of pro-fibrotic proteins by inducing RIPK1/RIPK3-mediated necroptosis in hematopoietic stem cells (HSCs). They also investigated knockdown of RIPK3 in HSCs and found that the effect of curcumol on attenuating hepatic fibrosis was impaired (73). Above all, necroptosis seems to play a positive role in the procession of fibrosis. In the meantime, other studies determined that caspase 8 was negatively correlated with liver fibrosis (63). To be specific, mice lacking the NLRP3 inflammasome had reduced liver fibrosis following $\mathrm{CCL}_{4}$ or thioacetamide treatment. Meanwhile, caspase 8 can inhibit the NLRP3 inflammasome. The activity of caspase 8 was inhibited when necroptosis started. Above all, we can deduce that liver fibrosis would accelerate when necroptosis occurs and necroptosis may be a basic factor in the pathogenesis of CLI. However, the relative potency of necroptosis to trigger CLI remains ill-defined.

\section{Caspase inbibitors are an attractive therapy for fibrotic liver disease}

Since necroptosis, which can be induced by inhibiting caspase- 8 activity, plays an important role in chronic liver diseases, some investigations were focused on caspases inhibitors. For instance, VX-166, an inhibitor of caspase, was used to inhibit apoptosis, which could significantly reduce liver damage and fibrosis, whereas it had no effect on the ALT/AST in the MCD model of NASH (88). In addition, the oral administration of the caspase inhibitor
GS-9450 (an inhibition of caspases 1, 8, and 9) and emricasan resulted in a decrease in alanine transaminase and $\alpha$-SMA levels in patients with NASH (89). Pan-caspase inhibitor IDN-6556 reduced the development of bile duct ligation-induced liver fibrosis (54). Despite that fact, emricasan treatment did not achieve the primary objective of ameliorating fibrosis in a large-scale randomized trial in NASH patients (90). These data demonstrate that caspase inhibitors may be an attractive antifibrotic therapy in NASH. However, is the protective effect of caspase inhibitors convincing? Is the protective effect of caspase inhibitors caused by necroptosis? The answers to these questions are still unclear. For faster development of a new strategy for liver disease treatment, the underlying mechanisms need further study.

\section{Necroptosis exerts both pro- and anti-tumoral effects during liver cancer}

HCC is the prevailing histological type of liver cancer, comprising approximately $80 \%$ of cases $(91,92)$. Cell death is a fundamental driver of liver diseases progression and the development of HCC (35). In tumors, tumor cells can usually evade apoptosis through a selection process (54). The step when parenchymal liver cells (hepatocytes and cholangiocytes) become insensitive to apoptotic cell death, is essential for malignant transformation. It should be noted that most human hepatoma cell lines such as Huh-7, HepG2, and Hep3B present a methylationdependent loss of RIPK 3 expression and thereby suppress the initiation of necroptosis, which indicates that escape from necroptosis is a similarly vital part in the malignant transformation of HCC. Furthermore, recovery of RIPK3 expression by demethylation of its promoter can restore the sensitivity of cell lines to chemotherapy, showing that regulating necroptosis may be an effective way to enhance the chemosensitivity of HCC, a tumor that generally is not responsive to traditional chemotherapy. The strategy of triggering necroptosis in cancer therapy shows great potential (21). Therefore, necroptosis can prevent tumor development when apoptosis fails to be induced. However, necroptosis can induce inflammatory responses and reportedly promotes cancer metastasis and immunosuppression. Additionally, even the same oncogenic driver will instigate different liver cancers, due to the different hepatic microenvironment. Aberrantly activated hepatocytes transformed into cholangiocarcinoma when embedded in a necroptosis-dominant hepatic 
microenvironment (93).

microRNAs (miRNAs or miRs) comprise a group of small noncoding RNAs that participate in cell proliferation, differentiation, apoptosis, and necroptosis $(94,95)$. The dysregulation of miRNAs has often been observed in HCC (96). The expression levels of Linc00176 is correlated both with the differentiation grade in primary HCCs and with the survival time of HCC patients by titrating tumor suppressor microRNAs. Linc00176depleted HepG2 cells experience necroptosis rather than apoptosis, as MLKL was detected in these cells (97). In addition, a study identified a novel three-miRNA signature, comprising miR-371-5p, miR-373 and miR-543, that appeared to orchestrate PCN in HCC by directly targeting the caspase-8 gene (Casp-8) (98). Hence, some kinds of miRNAs can modulate the process of tumorigenesis by way of necroptosis.

Collectively, increasing experimental evidence suggests that necroptosis pathways play critical roles in hepatocarcinogenesis, but the precise mechanism is less clear and still needs to be explored.

\section{Conclusions}

As a newly defined form of cell death, doubts still remain about the occurrence and role of necroptosis. Typically, the upregulation of RIPK1, RIPK3 and MLKL present the occurrence of necroptosis. However, detection of these molecular signals underlying necroptosis is somewhat difficult and has low specificity. There still are many controversies regarding this process that need to be addressed. However, the identification of necroptosis has changed our understanding about cell death and liver diseases. Based on current literature, it seems that necroptosis can play a vital role in different stages of liver disease. Since there are many challenges to clearly distinguish between necrosis, apoptosis, and necroptosis, it is necessary to find more specific canonical molecular signals in necroptosis to aid in its verification from different aspect. Moreover, it is recommended that further studies should focus on the role of necroptosis in different liver cells, including hepatocytes, cholangiocytes, and immune cells. Additionally, despite the unsatisfactory clinical trial results, pharmacological inhibition of RIPK1 emerges as a potential therapeutic method to cure chronic liver disease and HCC. Given that the role of RIPKs changes under different conditions, it is vital to clarify the underlying mechanism of necroptosis in different kinds of liver diseases. Furthermore, the contribution of RIPK3/MLKL remains debatable (11). Therefore, the effects of RIPK1, RIPK3, and MLKL should be studied separately and in greater detail. Most importantly, more specific downstream signaling molecules involved in necroptosis need to be detected.

\section{Acknowledgments}

Thanks for the language correction provided by EDITAGE. Funding: This work was supported by the Key Research \& Development Plan of Zhejiang Province (2019C04005), the major national $S \& T$ projects for infectious diseases (2018ZX10301401), the National Key Research and Development Program of China (2018YFC2000500).

\section{Footnote}

Reporting Checklist: The authors have completed the Narrative Review reporting checklist. Available at http:// dx.doi.org/10.21037/atm-20-5162

Peer Review File: Available at http://dx.doi.org/10.21037/ atm-20-5162

Conflicts of Interest: All authors have completed the ICMJE uniform disclosure form (available at http://dx.doi. org/10.21037/atm-20-5162). The authors have no conflicts of interest to declare.

Ethical Statement: The authors are accountable for all aspects of the work in ensuring that questions related to the accuracy or integrity of any part of the work are appropriately investigated and resolved.

Open Access Statement: This is an Open Access article distributed in accordance with the Creative Commons Attribution-NonCommercial-NoDerivs 4.0 International License (CC BY-NC-ND 4.0), which permits the noncommercial replication and distribution of the article with the strict proviso that no changes or edits are made and the original work is properly cited (including links to both the formal publication through the relevant DOI and the license). See: https://creativecommons.org/licenses/by-nc-nd/4.0/.

\section{References}

1. Wang FS, Fan JG, Zhang Z, et al. The global burden of liver disease: the major impact of China. Hepatology 
2014;60:2099-108.

2. Wang H, Lu Z, Zhao X. Tumorigenesis, diagnosis, and therapeutic potential of exosomes in liver cancer. J Hematol Oncol 2019;12:133.

3. Gong Y, Fan Z, Luo G, et al. The role of necroptosis in cancer biology and therapy. Mol Cancer 2019;18:100.

4. Yuan J, Amin P, Ofengeim D. Necroptosis and RIPK1mediated neuroinflammation in CNS diseases. Nat Rev Neurosci 2019;20:19-33.

5. Molnár T, Mazlo A, Tslaf V, et al. Current translational potential and underlying molecular mechanisms of necroptosis. Cell Death Dis 2019;10:860.

6. Pasparakis M, Vandenabeele P. Necroptosis and its role in inflammation. Nature 2015;517:311-20.

7. Li X, Yao X, Zhu Y, et al. The Caspase Inhibitor Z-VAD-FMK Alleviates Endotoxic Shock via Inducing Macrophages Necroptosis and Promoting MDSCsMediated Inhibition of Macrophages Activation. Front Immunol 2019;10:1824.

8. Kearney CJ, Martin SJ. An inflammatory perspective on necroptosis. Mol Cell 2017;65:965-73.

9. Newton K, Manning G. Necroptosis and inflammation. Annu Rev Biochem 2016;85:743-63.

10. Weinlich R, Oberst A, Beere HM, et al. Necroptosis in development, inflammation and disease. Nat Rev Mol Cell Biol 2017;18:127-36.

11. Kondylis V, Pasparakis M. RIP Kinases in Liver Cell Death, Inflammation and Cancer. Trends Mol Med 2019;25:47-63.

12. Schwabe RF, Luedde T. Apoptosis and necroptosis in the liver: a matter of life and death. Nat Rev Gastroenterol Hepatol 2018;15:738-52.

13. Guicciardi ME, Malhi H, Mott JL, et al. Apoptosis and necrosis in the liver. Compr Physiol 2013;3:977-1010.

14. Ray CA, Pickup DJ. The mode of death of pig kidney cells infected with cowpox virus is governed by the expression of the crmA gene. Virology 1996;217:384-91.

15. Degterev A, Huang Z, Boyce M, et al. Chemical inhibitor of nonapoptotic cell death with therapeutic potential for ischemic brain injury. Nat Chem Biol 2005;1:112-9.

16. Davidovich P, Kearney CJ, Martin SJ. Inflammatory outcomes of apoptosis, necrosis and necroptosis. Biol Chem 2014;395:1163-71.

17. Vandenabeele P, Declercq W, Van Herreweghe F, et al. The role of the kinases RIP1 and RIP3 in TNF-induced necrosis. Sci Signal 2010;3:re4.

18. Sun L, Wang H, Wang Z, et al. Mixed lineage kinase domain-like protein mediates necrosis signaling downstream of RIP3 kinase. Cell 2012;148:213-27.

19. Zhao J, Jitkaew S, Cai Z, et al. Mixed lineage kinase domain-like is a key receptor interacting protein 3 downstream component of TNF-induced necrosis. Proc Natl Acad Sci U S A 2012;109:5322-7.

20. Moriwaki K, Chan FK. Necroptosis-independent signaling by the RIP kinases in inflammation. Cell Mol Life Sci 2016;73:2325-34.

21. Qin X, Ma D, Tan YX, et al. The role of necroptosis in cancer: A double-edged sword? Biochim Biophys Acta Rev Cancer 2019;1871:259-66.

22. Shalini S, Dorstyn L, Dawar S, et al. Old, new and emerging functions of caspases. Cell Death Differ 2015;22:526-39.

23. Wang $\mathrm{Y}$, Zhao $\mathrm{M}, \mathrm{He} \mathrm{S}$, et al. Necroptosis regulates tumor repopulation after radiotherapy via RIP1/RIP3/MLKL/ JNK/IL8 pathway. J Exp Clin Cancer Res 2019;38:461.

24. Najafov A, Mookhtiar AK, Luu HS, et al. TAM Kinases Promote Necroptosis by Regulating Oligomerization of MLKL. Mol Cell 2019;75:457-68.e4.

25. Johnston A, Wang Z. Necroptosis: MLKL Polymerization. J Nat Sci 2018;4:e513.

26. Kaiser WJ, Sridharan H, Huang C, et al. Toll-like receptor 3-mediated necrosis via TRIF, RIP3, and MLKL. J Biol Chem 2013;288:31268-79.

27. Jun-Long H, Yi L, Bao-Lian Z, et al. Necroptosis Signaling Pathways in Stroke: From Mechanisms to Therapies. Curr Neuropharmacol 2018;16:1327-39.

28. Khoury MK, Gupta K, Franco SR, et al. Necroptosis in the Pathophysiology of Disease. Am J Pathol 2020;190:272-85.

29. Choi ME, Price DR, Ryter SW, et al. Necroptosis: a crucial pathogenic mediator of human disease. JCI Insight 2019; 4:e128834.

30. Vandenabeele P, Galluzzi L, Vanden Berghe T, et al. Molecular mechanisms of necroptosis: an ordered cellular explosion. Nat Rev Mol Cell Biol 2010;11:700-14.

31. Raninga PV, Di Trapani G, Vuckovic S, et al. Targeted knockdown of DJ-1 induces multiple myeloma cell death via KLF6 upregulation. Apoptosis 2016;21:1422-37.

32. Thakur B, Kumar Y, Bhatia A. Programmed necrosis and its role in management of breast cancer. Pathol Res Pract 2019;215:152652.

33. Jaeschke H, Ramachandran A, Chao X, et al. Emerging and established modes of cell death during acetaminopheninduced liver injury. Arch Toxicol 2019;93:3491-502.

34. He S, Huang S, Shen Z. Biomarkers for the detection of necroptosis. Cell Mol Life Sci 2016;73:2177-81. 
35. Schneider AT, Gautheron J, Feoktistova M, et al. RIPK1 Suppresses a TRAF2-Dependent Pathway to Liver Cancer. Cancer cell 2017;31:94-109.

36. Moriwaki K, Chan FK. The Inflammatory Signal Adaptor RIPK3: Functions Beyond Necroptosis. Int Rev Cell Mol Biol 2017;328:253-75.

37. Koo GB, Morgan MJ, Lee DG, et al. Methylationdependent loss of RIP3 expression in cancer represses programmed necrosis in response to chemotherapeutics. Cell Res 2015;25:707-25.

38. Roychowdhury S, McMullen MR, Pisano SG, et al. Absence of receptor interacting protein kinase 3 prevents ethanol-induced liver injury. Hepatology 2013;57:1773-83.

39. Cho YS, Challa S, Moquin D, et al. Phosphorylationdriven assembly of the RIP1-RIP3 complex regulates programmed necrosis and virus-induced inflammation. Cell 2009;137:1112-23.

40. Afonso MB, Rodrigues PM, Carvalho T, et al. Necroptosis is a key pathogenic event in human and experimental murine models of non-alcoholic steatohepatitis. Clin Sci (Lond) 2015;129:721-39.

41. Afonso MB, Rodrigues PM, Simao AL, et al. Activation of necroptosis in human and experimental cholestasis. Cell Death Dis 2016;7:e2390.

42. Li J, McQuade T, Siemer AB, et al. The RIP1/RIP3 necrosome forms a functional amyloid signaling complex required for programmed necrosis. Cell 2012;150:339-50.

43. Wree A, Holtmann TM, Inzaugarat ME, et al. Novel Drivers of the Inflammatory Response in Liver Injury and Fibrosis. Semin Liver Dis 2019;39:275-82.

44. Dara L. The Receptor Interacting Protein Kinases in the Liver. Semin Liver Dis 2018;38:73-86.

45. Krishna-Subramanian S, Singer S, Armaka M, et al. RIPK1 and death receptor signaling drive biliary damage and early liver tumorigenesis in mice with chronic hepatobiliary injury. Cell Death Differ 2019;26:2710-26.

46. Dixon LJ, Flask CA, Papouchado BG, et al. Caspase-1 as a central regulator of high fat diet-induced non-alcoholic steatohepatitis. PLoS One 2013;8:e56100.

47. Wang S, Pacher P, De Lisle RC, et al. A Mechanistic Review of Cell Death in Alcohol-Induced Liver Injury. Alcoholism, clinical and experimental research 2016;40:1215-23.

48. Barnes MA, Roychowdhury S, Nagy LE. Innate immunity and cell death in alcoholic liver disease: role of cytochrome P4502E1. Redox Biol 2014;2:929-35.

49. Han L, Teng Y, Fan Y, et al. Receptor-Interacting Protein Kinase 3 (RIPK3) mRNA Levels Are Elevated in Blood
Mononuclear Cells of Patients with Poor Prognosis of Acute-on-Chronic Hepatitis B Liver Failure. Tohoku J Exp Med 2019;247:237-45.

50. Günther C, He GW, Kremer AE, et al. The pseudokinase MLKL mediates programmed hepatocellular necrosis independently of RIPK3 during hepatitis. J Clin Invest 2016;126:4346-60.

51. Blériot C, Dupuis T, Jouvion G, et al. Liver-resident macrophage necroptosis orchestrates type 1 microbicidal inflammation and type-2-mediated tissue repair during bacterial infection. Immunity 2015;42:145-58.

52. Kang YJ, Bang BR, Han KH, et al. Regulation of NKT cell-mediated immune responses to tumours and liver inflammation by mitochondrial PGAM5-Drp1 signalling. Nat Commun 2015;6:8371.

53. Jouan-Lanhouet S, Arshad MI, Piquet-Pellorce C, et al. TRAIL induces necroptosis involving RIPK1/ RIPK3-dependent PARP-1 activation. Cell Death Differ 2012;19:2003-14.

54. Luedde T, Kaplowitz N, Schwabe RF. Cell death and cell death responses in liver disease: mechanisms and clinical relevance. Gastroenterology 2014;147:765-783.e4.

55. Stojanovic B, Milovanovic J, Arsenijevic A, et al. Galectin-3 Deficiency Facilitates TNF-alpha-Dependent Hepatocyte Death and Liver Inflammation in MCMV Infection. Front Microbiol 2019;10:185.

56. Wang H, Sun L, Su L, et al. Mixed lineage kinase domainlike protein MLKL causes necrotic membrane disruption upon phosphorylation by RIP3. Mol Cell 2014;54:133-46.

57. Dara L, Johnson H, Suda J, et al. Receptor interacting protein kinase 1 mediates murine acetaminophen toxicity independent of the necrosome and not through necroptosis. Hepatology 2015;62:1847-57.

58. Deutsch M, Graffeo CS, Rokosh R, et al. Divergent effects of RIP1 or RIP3 blockade in murine models of acute liver injury. Cell Death Dis 2015;6:e1759.

59. Li JX, Feng JM, Wang Y, et al. The B-Raf(V600E) inhibitor dabrafenib selectively inhibits RIP3 and alleviates acetaminophen-induced liver injury. Cell Death Dis 2014;5:e1278.

60. Ramachandran A, McGill MR, Xie Y, et al. Receptor interacting protein kinase 3 is a critical early mediator of acetaminophen-induced hepatocyte necrosis in mice. Hepatology 2013;58:2099-108.

61. Le Cann F, Delehouze C, Leverrier-Penna S, et al. Sibiriline, a new small chemical inhibitor of receptorinteracting protein kinase 1 , prevents immune-dependent hepatitis. FEBS J 2017;284:3050-68. 
62. Afonso MB, Rodrigues PM, Simao AL, et al. miRNA-21 ablation protects against liver injury and necroptosis in cholestasis. Cell Death Differ 2018;25:857-72.

63. Cubero FJ, Peng J, Liao L, et al. Inactivation of caspase 8 in liver parenchymal cells confers protection against murine obstructive cholestasis. J Hepatol 2018;69:1326-34.

64. Arshad MI, Piquet-Pellorce C, Filliol A, et al. The chemical inhibitors of cellular death, PJ34 and Necrostatin-1, down-regulate IL-33 expression in liver. J Mol Med (Berl) 2015;93:867-78.

65. Lawlor KE, Khan N, Mildenhall A, et al. RIPK3 promotes cell death and NLRP3 inflammasome activation in the absence of MLKL. Nat Commun 2015;6:6282.

66. Yoon S, Kovalenko A, Bogdanov K, et al. MLKL, the Protein that Mediates Necroptosis, Also Regulates Endosomal Trafficking and Extracellular Vesicle Generation. Immunity 2017;47:51-65.e7.

67. Wong WW, Vince JE, Lalaoui N, et al. cIAPs and XIAP regulate myelopoiesis through cytokine production in an RIPK1- and RIPK3-dependent manner. Blood 2014;123:2562-72.

68. Hänggi K, Vasilikos L, Valls AF, et al. RIPK1/RIPK3 promotes vascular permeability to allow tumor cell extravasation independent of its necroptotic function. Cell Death Dis 2017;8:e2588.

69. Stauffer JK, Scarzello AJ, Jiang Q, et al. Chronic inflammation, immune escape, and oncogenesis in the liver: a unique neighborhood for novel intersections. Hepatology 2012;56:1567-74.

70. Xiao M, Chen W, Wang C, et al. Senescence and cell death in chronic liver injury: roles and mechanisms underlying hepatocarcinogenesis. Oncotarget 2017;9:8772-84.

71. Gautheron J, Vucur M, Reisinger F, et al. A positive feedback loop between RIP3 and JNK controls non-alcoholic steatohepatitis. EMBO Mol Med 2014;6:1062-74.

72. Zhang Z, Xie G, Liang L, et al. RIPK3-Mediated Necroptosis and Neutrophil Infiltration Are Associated with Poor Prognosis in Patients with Alcoholic Cirrhosis. J Immunol Res 2018;2018:1509851.

73. Jia Y, Wang F, Guo Q, et al. Curcumol induces RIPK1/ RIPK3 complex-dependent necroptosis via JNK1/2ROS signaling in hepatic stellate cells. Redox Biol 2018;19:375-87.

74. Upton JW, Kaiser WJ, Mocarski ES. Virus inhibition of RIP3-dependent necrosis. Cell Host Microbe 2010;7:302-13.
75. Inoue $J$, Ninomiya $M$, Shimosegawa $T$, et al. Cellular Membrane Trafficking Machineries Used by the Hepatitis Viruses. Hepatology 2018;68:751-62.

76. Lim EJ, El Khobar K, Chin R, et al. Hepatitis C virusinduced hepatocyte cell death and protection by inhibition of apoptosis. J Gen Virol 2014;95:2204-15.

77. Zhou Y, Jin H, Wu Y, et al. Gallic acid protects against ethanol-induced hepatocyte necroptosis via an NRF2-dependent mechanism. Toxicol In Vitro : an international journal published in association with BIBRA 2019;57:226-32.

78. Vucur M, Reisinger F, Gautheron J, et al. RIP3 inhibits inflammatory hepatocarcinogenesis but promotes cholestasis by controlling caspase-8- and JNK-dependent compensatory cell proliferation. Cell Rep 2013;4:776-90.

79. Ren Y, Su Y, Sun L, et al. Discovery of a Highly Potent, Selective, and Metabolically Stable Inhibitor of ReceptorInteracting Protein 1 (RIP1) for the Treatment of Systemic Inflammatory Response Syndrome. J Med Chem 2017;60:972-86.

80. Majdi A, Aoudjehane L, Ratziu V, et al. Inhibition of receptor-interacting protein kinase 1 improves experimental non-alcoholic fatty liver disease. J Hepatol 2020;72:627-35.

81. Wu X, Nagy LE. MLKL contributes to Western dietinduced liver injury through inhibiting autophagy. Autophagy 2020;16:1351-2.

82. Wu X, Poulsen KL, Sanz-Garcia C, et al. MLKLdependent signaling regulates autophagic flux in a murine model of non-alcohol-associated fatty liver and steatohepatitis. J Hepatol 2020;73:616-27.

83. Roychowdhury S, McCullough RL, Sanz-Garcia C, et al. Receptor interacting protein 3 protects mice from high-fat diet-induced liver injury. Hepatology 2016;64:1518-33.

84. Chenxu G, Minxuan X, Yuting Q, et al. Loss of RIP3 initiates annihilation of high-fat diet initialized nonalcoholic hepatosteatosis: A mechanism involving Tolllike receptor 4 and oxidative stress. Free Radic Biol Med 2019;134:23-41.

85. Longhi R, Almeida RF, Machado L, et al. Effect of a trans fatty acid-enriched diet on biochemical and inflammatory parameters in Wistar rats. Eur J Nutr 2017;56:1003-16.

86. Zhao X, Shen C, Zhu H, et al. Trans-Fatty Acids Aggravate Obesity, Insulin Resistance and Hepatic Steatosis in C57BL/6 Mice, Possibly by Suppressing the IRS1 Dependent Pathway. Molecules 2016;21:705.

87. Choi HS, Kang JW, Lee SM. Melatonin attenuates carbon tetrachloride-induced liver fibrosis via inhibition of 
necroptosis. Transl Res 2015;166:292-303.

88. Witek RP, Stone WC, Karaca FG, et al. Pan-caspase inhibitor VX-166 reduces fibrosis in an animal model of nonalcoholic steatohepatitis. Hepatology 2009;50:1421-30.

89. Barreyro FJ, Holod S, Finocchietto PV, et al. The pancaspase inhibitor Emricasan (IDN-6556) decreases liver injury and fibrosis in a murine model of non-alcoholic steatohepatitis. Liver Int 2015;35:953-66.

90. Harrison SA, Goodman Z, Jabbar A, et al. A randomized, placebo-controlled trial of emricasan in patients with NASH and F1-F3 fibrosis. J Hepatol 2020;72:816-27.

91. Mazzoccoli G, Miele L, Marrone G, et al. A Role for the Biological Clock in Liver Cancer. Cancers (Basel) 2019;11:1778.

92. Cho K, Ro SW, Seo SH, et al. Genetically Engineered Mouse Models for Liver Cancer. Cancers (Basel) 2019;12:14.

93. Seehawer M, Heinzmann F, D'Artista L, et al. Necroptosis microenvironment directs lineage commitment in liver

Cite this article as: Li X, Dong G, Xiong H, Diao H. A narrative review of the role of necroptosis in liver disease: a double-edged sword. Ann Transl Med 2021;9(5):422. doi: 10.21037/atm-20-5162 cancer. Nature 2018;562:69-75.

94. Xie KL, Zhang YG, Liu J, et al. MicroRNAs associated with $\mathrm{HBV}$ infection and HBV-related HCC. Theranostics 2014;4:1176-92.

95. Boursier J, Guillaume M, Leroy V, et al. New sequential combinations of non-invasive fibrosis tests provide an accurate diagnosis of advanced fibrosis in NAFLD. J Hepatol 2019;71:389-96.

96. Musaddaq G, Shahzad N, Ashraf MA, et al. Circulating liver-specific microRNAs as noninvasive diagnostic biomarkers of hepatic diseases in human. Biomarkers 2019;24:103-9.

97. Tran DDH, Kessler C, Niehus SE, et al. Myc target gene, long intergenic noncoding RNA, Linc00176 in hepatocellular carcinoma regulates cell cycle and cell survival by titrating tumor suppressor microRNAs. Oncogene 2018;37:75-85.

98. Visalli M, Bartolotta M, Polito F, et al. miRNA expression profiling regulates necroptotic cell death in hepatocellular carcinoma. Int J Oncol 2018;53:771-80. 